\title{
Desar rollo y evaluación de suplementos alimenticios para el Programa de Educación, Salud y Alimentación
}

Jorge L. Rosado, M. en C., PhD., (1) Juan Rivera, M. en C., PhD., (2) G ladys López, Q uím., ${ }^{(1)}$

Lourdes Solano, Lic. en N utr., ${ }^{(1)}$ Guadalupe Rodríguez, Lic. en N utr., (2) Esther Casanueva, Lic. en N utr., ${ }^{(3)}$

Alberto García-Aranda, M.C. ${ }^{\left({ }^{(4)}\right.}$ Georgina Toussaint, Lic en N utr., ${ }^{(4)}$ Irene Maulen, M.C. ${ }^{(5)}$

Rosado JL, Rivera J, López G, Solano L, Rodríguez G, Casanueva E, García-A randa A, Toussaint G, Maulen I.

Desarrollo y evaluación de suplementos alimenticios para el Programa de Educación, Salud y Alimentación. Salud Publica Mex 1999;41:153-162.

\section{Resumen}

Objetivo. D esarrollar y evaluar suplementos alimenticios destinados a un programa de asistencia social. Material y métodos Para desar rollar los suplementos alimenticios se establecieron criterios de composición, propiedades fisicoquímicas, así como de facilidad de producción y utilización. Los productos desarrollados se evaluaron inicialmente mediante pruebas sensoriales de nivel de agrado comparativas en 40 niños, 52 mujeres embarazadas y 62 mujeres en periodo de lactancia; posteriormente, se hicieron estudios de aceptación y consumo en 108 niños y 128 mujeres de una comunidad rural del estado de Morelos. Resultados. Se presentan las fórmulas específicas y los procesos para la elaboración de los suplementos alimenticios. En las evaluaciones sensoriales los productos fueron ampliamente aceptados, de tal manera que se presentaron calificaciones promedio de entre 4.11 y 4.29 en la bebida para niños y de entre 3.98 y 4.15 en la papilla (calificación de 1 a 5). Los productos para mujeres recibieron calificaciones promedio de entre 4.75 y 5.70 en las embarazadas y de entre 4.8 y 5.4 en las que estaban en periodo de lactancia (calificación de 1 a 7). En el estudio en comunidad los suplementos fueron ampliamente aceptados en general. Los consumos promedio fueron $>75 \%$ de lo ofrecido en los niños y $>98 \%$ de lo
Rosado JL, Rivera J, López G, Solano L, Rodríguez G, Casanueva E, García-A randa A, Toussaint G, Maulen I.

Development and evaluation of nutritional supplements for the Program on Education, Health and Nutrition.

Salud Publica Mex 1999;41:153-162.

\begin{abstract}
A bstract
Objective.To develop and evaluate nutritional supplements destined to a program of social assistance. Material and methods In the design of the nutritional supplements a series of criteria were considered including nutrient composition, physicochemical properties and feasibility of production and utilization. Final products were initially evaluated to determine the level of acceptance in 40 children, 52 pregnant wo men and 62 lactating women in Mexico C ity. A community trial was also carried out to determine acceptance and consumption in 108 children and 128 women from a rural community in the state of Morelos. Results. The specific formulation and technical processes of production of the nutritional supplements are presented. Products proved to be widely accepted, with average scores of 4.11-4.29 for the children's beverage, and 3.98-4.15 for a more viscous pap (range of scores was 1 to 5). Products for women received average scores from 4.75 to 5.70 in pregnant and from 4.8 to 5.4 in lactating women (range of scores from 1 to 7). In the community trial, supplements were very well accepted. Average consumption was $>75 \%$ among children and $>98 \%$ among women. Mean energy intake from supplements was $244 \mathrm{~K} \mathrm{cal} /$ day for women, and for children, 168 $\mathrm{K}$ cal/day with the pap and $147 \mathrm{Kcal} /$ day with the beverage.
\end{abstract}

(1) Departamento de Fisiología de la N utrición, Instituto N acional de la N utrición Salvador Zubirán, México.

(2) Instituto $\mathrm{N}$ acional de Salud Pública, Cuernavaca, Morelos, México.

(3) Instituto $N$ acional de Perinatología, México.

(4) Hospital Infantil de México Federico Gómez, México.

(5) Instituto Nacional de Pediatría, México.

Fecha de recibido: 20 de mayo de 1998 - Fecha de aprobado: 5 de octubre de 1998

Solicitud de sobretiros: Dr. Jorge L. Rosado. Instituto N acional de la N utrición Salvador Zubirán. Vasco de Q uiroga N 0. 15,Tlalpan, 14000 México, D.F. Correo electrónico: rosado@ servidor.unam.mx 
ofrecido en las mujeres. En promedio las mujeres recibieron $244 \mathrm{Kcal} /$ día, y los niños, $168 \mathrm{Kcal} /$ día con la papilla y $147 \mathrm{Kcal} /$ día con la bebida; en to dos los casos se observó consistencia en el consumo a lo largo del tiempo. Conclusiones. Se desarrollaron y evaluaron nueve suplementos alimenticios que cumplen con las características nutricias, fisico químicas e higiénicas adecuadas para la población objetivo, además de que su elaboración es relativamente sencilla y resultan ampliamente aceptados.

Palabras clave: alimentos fortificados; micronutrientes; trastornos nutricionales; crecimiento; embarazo; lactancia materna; México
Consumption was consistent in all cases along the study. Conclusions. N ine nutritrional supplements were developed and evaluated which comply with the necessary nutritional, physicochemical and hygienic characteristics for the target population, besides being relatively simple to prepare, and widely well accepted and consumed.

Key words: food, fortified; micronutrients; nutrition disorders; growth; pregnancy; breast feeding; Mexico
E xiste evidencia documentada de desnutrición por deficiencia de micronutrimentos entre la población de México; ${ }^{1-4}$ este padecimiento afecta principalmente a niños menores de cinco años y a mujeres gestantes y en periodos de lactancia. Una de las formas de atender la desnutrición por deficiencia de micronutrimentos en los grupos de población con alto riesgo es, justamente, mediante la suplementación de dichos nutrimentos. Existen varios programas de suplementación alimenticia que han tenido éxito en la atención y el mejoramiento del estado nutricio, ${ }^{5-7}$ entre los cuales se encuentran los programas controlados ${ }^{5,6}$ y los de gran escala como el de Tamil Nadu en la India. ${ }^{7}$

Si bien el éxito de un programa depende de muchos factores, ${ }^{8}$ el hecho de contar con un suplemento cuya composición de nutrimentos se ajuste a las necesidades de la población objetivo y cuya aceptación clínica y sensorial sea lo más generalizada posible, puede contribuir esencialmente a mejorar el estado de nutrición de la población a la que está destinado. Existen ejemplos de programas de suplementación alimenticia en México que han fracasado debido, sobre todo, a que el suplemento no era ampliamente aceptado o a que su composición era inapropiada para la población objetivo. ${ }^{9}$

Entre los factores más importantes que deben considerarse están los asociados con la calidad nutricia y la composición del suplemento. El producto debe contener todos los nutrimentos en cantidades adecuadas según la edad, el estado fisiológico y el estado nutricio de la población a la que va dirigido. Los nutrimentos deben encontrarse en forma altamente biodisponible y deben evitarse, en lo posible, sustancias o componentes que interfieran con la absorción y la utilización adecuada de los nutrimentos en el suplemento. En caso de que dichas sustancias se en- cuentren en el producto, deben conocerse cuantitativamente para poder determinar la proporción de nutrimento biodisponible.

Por otra parte, el suplemento debe ser tolerado clínicamente de tal manera que su ingestión en las cantidades recomendadas no cause problemas de intolerancia o mala absorción, además de que debe ser ampliamente aceptado desde el punto de vista sensorial. La falta de atención a este último aspecto ha hecho fracasar a ciertos programas de suplementación en México. ${ }^{9}$ El producto debe tener muy buen sabor, ser fácil de preparar y consumir y de apariencia atractiva, de manera que la población objetivo lo consuma habitualmente y con la frecuencia recomendable. Es muy deseable que el suplemento sea lo bastante atractivo como para que el individuo al que está destinado lo demande y lo consuma no sólo por sus efectos benéficos para la salud, sino porque además le gusta.

El suplemento debe cumplir con una serie de propiedades fisicoquímicas que permitan la facilidad de su uso, su estabilidad y su durabilidad por un periodo suficiente desde que se produce hasta que se consume. Así por ejemplo, si se trata de un producto líquido, deberá ser homogéneo, de baja viscosidad relativa y estéril; si su presentación es en forma de polvo, deberá tener una solubilidad elevada y libre de microrganismos patógenos.

Por último, es deseable que el suplemento cumpla con una serie de características que faciliten su utilización y obtención al menor costo posible; al respecto se debe cuidar que los ingredientes estén disponibles en el lugar donde se va a fabricar el producto, que el proceso de elaboración sea lo más sencillo y barato posible, que la presentación o forma del producto se ajuste al mecanismo de distribución disponible y a las características de la población a la que está dirigi- 
do el programa, y que la presentación y el empaque sean compatibles con la vida útil que se requiere en el producto.

En este estudio se abordan los aspectos de desarrollo y evaluación de suplementos alimenticios destinados a un programa de asistencia social, entre cuyos objetivos está el de mejorar el estado de nutrición de los niños de 4 a 24 meses de edad y de las mujeres embarazadas y en periodo de lactancia.

\section{Material y métodos}

\section{Características del suplemento alimenticio}

Antes de considerar los ingredientes y la composición de los suplementos, se definió una serie de características con las que deberían cumplir los mismos: a) los suplementos deberían aportar los nutrimentos que se sabe son deficientes en la población mexicana y las cantidades que se recomiendan para llevar una dieta adecuada; b) los ingredientes seleccionados para su elaboración deberían ser de fácil adquisición en México y de bajo costo relativo; c) el proceso involucrado en la elaboración de los productos debería ser lo más sencillo y de menor costo posible; d) el sabor debería ser agradable para estimular el consumo de los suplementos; e) la forma final de los productos debería ser práctica para facilitar su distribución y consumo y, finalmente, f) la presentación y el empaque de los productos deberían ser atractivos y permitir la conservación adecuada del producto por un lapso razonable.

\section{Composición nutrimental}

Para determinar la composición nutrimental del suplemento se revisaron algunos de los documentos más relevantes sobre las deficiencias de nutrimentos que se presentan más comúnmente en México. ${ }^{1-4,10,11}$ Como resultado de este análisis se concluyó que el suplemento debía contribuir con la ingestión de proteína de alta calidad, de energía y de las vitaminas y los minerales cuya deficiencia en algunos grupos de población del país está documentada. Para los niños, en particular, se recomienda $5.8 \mathrm{~g}$ de proteína, $194 \mathrm{Kcal}$ de energía, $10 \mathrm{mg}$ de hierro, $10 \mathrm{mg}$ de zinc, $400 \mathrm{mcg}$ de vitamina $\mathrm{A}, 6 \mathrm{mg}$ de vitamina $\mathrm{E}, 40 \mathrm{mg}$ de vitamina $\mathrm{C}$, $0.8 \mathrm{mg}$ de vitamina $B_{2^{\prime}} 0.7$ mcg de vitamina $B_{12}$ y 50 mcg de ácido fólico. Para las mujeres, la composición de los suplementos debe ser la siguiente: $12-15 \mathrm{~g}$ de proteína, $250 \mathrm{Kcal}$ de energía, $15 \mathrm{mg}$ de hierro, $15 \mathrm{mg}$ de zinc, $10 \mathrm{mg}$ de vitamina E, 70 miligramos de vitamina $C, 2.6 \mathrm{mcg}$ de vitamina $B_{12^{\prime}} 100$ mcg de ácido fólico y 100 mcg de yodo.

\section{Selección de ingredientes y su procesamiento}

Se decidió incluir leche entera como la principal fuente de proteínas y de energía de los suplementos dadas sus propiedades nutricias y funcionales. La leche posee una densidad energética elevada, especialmente si es entera; el aporte de proteína de alta calidad es también elevado, además de ser una fuente importante de algunos minerales y vitaminas como calcio, fósforo y riboflavina. La lactosa es el carbohidrato cuantitativamente más importante en la leche y en ciertos productos lácteos. En algunos estudios se ha sugerido que el contenido de lactosa de la leche puede constituir una limitante para su utilización en programas de suplementación alimenticia debido a la alta incidencia de deficiencia de lactasa que se había notificado en el país hasta hace algunos años. ${ }^{12,13} \mathrm{Sin}$ embargo, mediante estudios más recientes realizados en diferentes regiones de México, en los que se han involucrado individuos de 0 a 99 años, tanto de zonas rurales como urbanas del país, ${ }^{14-16}$ se ha llegado a la conclusión de que la deficiencia de lactasa y la intolerancia a la lactosa no constituyen un problema que limite de manera significativa el consumo de leche en México; por lo tanto, el uso de la leche en programas de asistencia social no debe limitarse con base en la existencia de intolerancia a la lactosa. ${ }^{17,18}$ Por estas razones, se decidió incluir a la leche como base para la elaboración de los suplementos alimenticios.

Con la finalidad de alcanzar las especificaciones de energía que se habían propuesto, se adicionó almidón de maíz hidrolizado con un equivalente de dextrosa de 20; de esta manera se adiciona energía sin causar un impacto importante en la viscosidad y tampoco se produce un incremento significativo en la osmolaridad. Con el objeto de lograr un nivel de dulzor suficiente para obtener un sabor agradable, se incluyó una cantidad mínima de azúcar. Los sabores que se seleccionaron fueron plátano, vainilla y chocolate para el suplemento de los niños, y plátano, vainilla y sin saborizante (sabor natural) para el de las mujeres embarazadas y en periodo de lactancia.

Una vez seleccionados los ingredientes apropiados, se decidió que los suplementos fueran en polvo para reducir su costo y facilitar su distribución. De esa 
manera se pudo optar por un proceso muy sencillo de mezclado de ingredientes en polvo, lo cual hizo posible abaratarlos aún mas.

\section{Pruebas de evaluación sensorial}

A fin de que los suplementos fueran ampliamente aceptados, durante su desarrollo se realizaron evaluaciones sensoriales en 40 niños que asistían a la Guardería No. 53439 del ISSSTE, en 53 mujeres gestantes y 62 mujeres en periodo de lactancia, todas pacientes de consulta externa del Hospital Manuel Gea González. A cada uno de los niños y de las mujeres se les aplicaron pruebas de nivel de agrado con una escala hedónica de 1 a 5 en los niños y de 1 a 7 en las mujeres, ${ }^{19}$ y se compararon con un producto comercialmente disponible y altamente aceptado (Compañía Nestlé S.A. de C.V. Quick M.R.). En el caso de la papilla fue imposible compararla con productos comerciales apropiados debido a la inexistencia de estos últimos. Los saborizantes se desarrollaron para llenar este requisito de sabor y aceptabilidad. En el caso de los niños, encuestadores entrenados aplicaron el instrumento, calificando la respuesta sensorial del niño según su comportamiento y expresión, de acuerdo con metodologías validadas. ${ }^{19}$

\section{Estudios de aceptación y consumo de los suplementos en comunidad}

El estudio se realizó en Xoxocotla, en el estado de Morelos, México. Se trata de una comunidad semirural con una población aproximada de 16000 habitantes y una superficie territorial de $7.5 \mathrm{~km}^{2}$. Está ubicada a una altitud de $930 \mathrm{msnm}$ y el clima es cálido semihúmedo con temperatura media anual de 26 a $30{ }^{\circ} \mathrm{C}$. La actividad principal es la agricultura y hay una alta prevalencia de familias en extrema pobreza.

Se estudiaron niños menores de tres años, mujeres embarazadas y mujeres en periodo de lactancia. El primer grupo se integró con 108 niños; incluyó a 58 lactantes y a 23 destetados, todos menores de dos años de edad y con diversos niveles de nutrición, así como a 27 niños destetados, de entre 2 y 3 años de edad, con algún grado de desnutrición, esto es, con un peso/edad menor a -1 desviación estándar (DE) de acuerdo con el patrón de referencia de la Organización Mundial de la Salud (OMS). ${ }^{20}$ El grupo de 128 mujeres se integró con 64 embarazadas y 64 en periodo de lactancia.

$\mathrm{Al}$ inicio del estudio los niños y las mujeres fueron pesados y medidos por encuestadores debidamente estandarizados. Esta información se utilizó para selec- cionar a los niños desnutridos mayores de dos años y como referencia para los grupos restantes.

Los grupos estudiados recibieron una ración diaria de los productos desarrollados, mismos que se prepararon de acuerdo con la descripción correspondiente que se hace en la sección de resultados. Los productos se pesaron en una báscula con precisión de $2 \mathrm{~g}$ (OHAUS, México, D.F.). Una encuestadora capacitada registró la cantidad servida y el residuo no consumido, y determinó por diferencia la cantidad ingerida, la que se expresó también como proporción de la porción servida.

Se realizaron dos tipos de evaluación: a) una prueba de aceptación de los productos y b) una evaluación de las cantidades consumidas a lo largo de dos semanas de seguimiento, durante las cuales las mujeres y los niños acudieron a un centro de suplementación localizado a una distancia accesible de sus hogares. En casos excepcionales, el producto fue llevado al domicilio por promotoras capacitadas, quienes seguían los procedimientos de registro de consumo antes descritos.

La prueba de aceptación en los niños se llevó a cabo de la siguiente manera: durante tres días consecutivos la madre ofreció a su hijo una ración de papilla, cada día, de distinto sabor, y en los tres días siguientes se hizo lo mismo con la bebida. El orden para la administración de los diferentes sabores se estableció aleatoriamente. En el caso de las mujeres la prueba de aceptación de la bebida se realizó durante tres días consecutivos, asignando también cada uno de los diferentes sabores diariamente en forma aleatoria.

La fase de seguimiento o evaluación al consumo se realizó durante un periodo de 14 días consecutivos (exceptuando domingos), en los cuales se ofreció el producto de consistencia y sabor preferido por las madres y sus hijos durante la fase de aceptación. En los casos de asistencia irregular, se realizaron visitas domiciliarias para indagar acerca de las razones por las cuales la madre o el niño no habían acudido al centro de suplementación; en su caso, se comentaron aspectos relacionados con el producto que pudieran interferir con su aceptación.

Se analizaron las diferencias en el consumo según los diferentes sabores, tanto para los resultados de la prueba de aceptación como para los del periodo de seguimiento. Para fines del análisis, los niños del estudio se dividieron en tres grupos: a) menores de dos años que continuaban recibiendo leche materna $(<2$ años lactantes); b) menores de dos años que ya no recibían leche materna ( $<2$ años destetados), y c) niños de entre 2 y 3 años de edad con un peso/edad $<-1 \mathrm{DE}$ según el patrón de referencia de la Organiza- 
ción Mundial de la Salud (> 2 años desnutridos). En el caso de las mujeres, para fines de análisis se formaron dos grupos: a) embarazadas y b) en periodo de lactancia.

El tratamiento estadístico de los datos se realizó mediante análisis de varianza, utilizando la prueba de comparaciones múltiples de Scheffé, así como prueba de $t$ para evaluar diferencias de medias en el consumo de acuerdo con la consistencia del producto, el sabor y el grupo de estudio, tanto en la fase de aceptación como durante la de seguimiento.

\section{Resultados}

\section{Definición de fórmulas y proceso de fabricación}

Las fórmulas de los suplementos desarrollados se muestran en el cuadro I. Los productos para niños se desarrollaron en dos versiones: una para preparar bebida y otra para preparar papilla en sabores de chocolate, plátano y vainilla. Los productos para mujeres embarazadas y en periodo de lactancia se desarrollaron sólo como bebida en sabores de vainilla y plátano, aunque también se elaboró una tercera versión sin saborizante (sabor natural).

El proceso para la elaboración de los productos consiste básicamente en mezclar los ingredientes en seco y empacarlos en la opción que se juzgue más conveniente (p.e., sobres laminados, lata de lámina o cartón, bolsa de polietileno, etc.) A continuación se especifican los pasos que deben seguirse: a) pesado de los ingredientes, que se hace en la forma convencional respetando la precisión requerida según el tamaño del lote que se vaya a preparar; b) elaboración de premezclas: con la finalidad de hacer más eficiente el mezclado de los ingredientes, los sabores, las vitaminas y los minerales se adicionan a una base de almidón de maíz hidrolizado antes de adicionarse al mezclador, haciendo una premezcla lo más homogénea posible (ésta puede hacerse en un mezclador más pequeño o en una bolsa de plástico previamente sanitizada); c) mezclado de ingredientes: en primer lugar, se adiciona la leche en polvo, después el almidón de maíz hidrolizado, enseguida la premezcla de almidón de maíz hidrolizado, saborizantes, vitaminas y minerales y, por último, se adiciona el azúcar. Se recomienda utilizar algún sistema que garantice un mezclado eficaz de los ingredientes. El tiempo destinado a esta operación deberá ajustarse al tipo de mezclador que se utilice y al tamaño del lote que se prepare.

\section{Formas de uso de los suplementos}

Para lograr una bebida y/o papilla con el aporte nutricio y con las características sensoriales y funcionales requeridas, la dosis necesaria es de 44 gramos de los productos para niños y de 52 gramos del producto para mujeres. El suplemento tipo bebida para niños se prepara disolviendo la dosis en 150 mililitros de agua (un vaso pequeño) y mezclando con una cuchara. Si se utilizan los ingredientes apropiados el producto se disuelve fácilmente con agitación; la mezcla debe consumirse inmediatamente. El suplemento tipo papilla para niños se logra disolviendo la dosis mencionada en 25 mililitros de agua y mezclando con una cuchara hasta obtener una solución homogénea. Este producto se disuelve fácilmente con agitación y debe consumirse inmediatamente. La bebida para mujeres se logra disolviendo la dosis establecida en 150 mililitros de agua (un vaso pequeño) y mezclando con una cuchara hasta obtener una solución homogénea. El suplemento también se disuelve fácilmente con agitación y debe consumirse de inmediato.

\section{Cuadro I}

\section{Fórmulas obtenidas para los SUPLementos alimenticios. MéXico, 1998}

\begin{tabular}{|c|c|c|c|c|c|c|c|c|c|}
\hline \multirow[b]{2}{*}{ Ingredientes } & \multicolumn{3}{|c|}{ Bebida para niños } & \multicolumn{3}{|c|}{ Papilla para niños } & \multicolumn{3}{|c|}{$\begin{array}{c}\text { Bebida para mujeres embarazadas } \\
\text { y en periodo de lactancia }\end{array}$} \\
\hline & $\begin{array}{c}\text { Sabor } \\
\text { chocolate } \\
\mathrm{g} / 100 \mathrm{~g}\end{array}$ & $\begin{array}{l}\text { Sabor } \\
\text { vainilla } \\
\mathrm{g} / 100 \mathrm{~g}\end{array}$ & $\begin{array}{c}\text { Sabor } \\
\text { plátano } \\
\text { g/100 g }\end{array}$ & $\begin{array}{c}\text { Sabor, } \\
\text { chocolate } \\
\mathrm{g} / 100 \mathrm{~g}\end{array}$ & $\begin{array}{c}\text { Sabor } \\
\text { vainilla } \\
\mathrm{g} / 100 \mathrm{~g}\end{array}$ & $\begin{array}{c}\text { Sabor } \\
\text { plátano } \\
\text { g/100 g }\end{array}$ & $\begin{array}{l}\text { Sabor } \\
\text { natural } \\
\mathrm{g} / 100 \mathrm{~g}\end{array}$ & $\begin{array}{l}\text { Sabor } \\
\text { vainilla } \\
\mathrm{g} / 100 \mathrm{~g}\end{array}$ & $\begin{array}{c}\text { Sabor } \\
\text { plátano } \\
\text { g/100 g }\end{array}$ \\
\hline Leche entera en polvo & 54.93 & 54.93 & 54.93 & 54.93 & 54.93 & 54.93 & 80.0 & 80.00 & 80.0 \\
\hline Azúcar & 7.95 & 7.95 & 7.95 & 5.68 & 4.55 & 4.55 & 9.61 & 9.61 & 9.61 \\
\hline Maltodextrina & 34.30 & 35.23 & 36.70 & 37.25 & 39.75 & 40.17 & 10.01 & 8.68 & 9.89 \\
\hline Vitaminas y minerales & 0.32 & 0.32 & 0.32 & 0.32 & 0.32 & 0.3217 & 0.38 & 0.38 & 0.38 \\
\hline Sabor chocolate & 2.5 & - & - & 1.82 & - & - & - & - & - \\
\hline Sabor vainilla & - & 1.57 & - & - & 0.45 & - & - & 1.33 & - \\
\hline Sabor plátano & - & - & 0.10 & - & - & 0.034 & - & - & 0.12 \\
\hline
\end{tabular}




\section{Resultados de la evaluación sensorial}

En el cuadro II se describen las calificaciones que se obtuvieron con las mujeres embarazadas y con aquellas en periodo de lactancia, durante las pruebas de evaluación sensorial de los suplementos alimenticios. El sabor de plátano resultó con una calificación significativamente más alta que los de vainilla y natural entre las mujeres embarazadas, en tanto que en aquellas en periodo de lactancia no hubo diferencia estadística entre los tres sabores. En ambos grupos el producto comercial sabor vainilla resultó con un nivel de agrado estadísticamente menor que el sabor vainilla del producto desarrollado. En el cuadro II se muestran también las calificaciones que se obtuvieron con los niños en las pruebas de evaluación sensorial de la bebida y de la papilla. No hubo diferencia significativa en el nivel de agrado entre los diferentes sabores y los resultados fueron similares a los obtenidos con los productos comerciales. En el caso de las papi-

\section{Cuadro II \\ Calificación de nivel de agrado obtenido EN LA EVALUACIÓN SENSORIAL De LOS SUPLementos Alimenticios. MéXICO, 1998}

Producto evaluado

Calificación $(\bar{X} \pm D E)$

Bebida para mujer embarazada

\begin{tabular}{ll} 
Sabor vainilla & $4.75 \pm 2.12^{\mathrm{a}}$ \\
\hline Sabor plátano & $5.70 \pm 1.50^{\mathrm{b}}$ \\
\hline Sabor natural & $4.75 \pm 2.00^{\mathrm{a}}$ \\
\hline Comercial sabor vainilla & $3.13 \pm 2.19^{\mathrm{c}}$
\end{tabular}

Bebida para mujer en periodo de lactancia

Sabor vainilla $\quad 5.29 \pm 1.93^{\circ}$

\begin{tabular}{ll} 
Sabor plátano & $5.48 \pm 2.04^{\mathrm{a}}$ \\
\hline Sabor natural & $4.84 \pm 2.11^{\mathrm{a}}$ \\
\hline Comercial sabor vainilla & $3.45 \pm 2.86^{\mathrm{b}}$
\end{tabular}

Bebida para niño

Sabor vainilla $\quad 4.26 \pm 0.88^{a}$

Sabor plátano $\quad 4.11 \pm 0.99^{\mathrm{a}, \mathrm{b}}$

Sabor chocolate $\quad 4.29 \pm 0.85^{\mathrm{a}, \mathrm{b}}$

Comercial sabor vainilla $\quad 3.94 \pm 1.19^{\mathrm{a}}$

Comercial sabor chocolate $\quad 4.38 \pm 0.83^{\mathrm{b}}$

\begin{tabular}{ll}
$\begin{array}{l}\text { Papilla para niño } \\
\text { Sabor vainilla }\end{array}$ & $3.98 \pm 1.01^{\mathrm{a}, \mathrm{b}}$ \\
\hline Sabor plátano & $3.67 \pm 1.20^{\mathrm{a}}$ \\
\hline Sabor chocolate & $4.15 \pm 0.96^{\mathrm{b}}$
\end{tabular}

Nota: Los promedios con diferentes superíndices dentro del mismo producto son estadísticamente diferentes $(p<0.05)$ llas, la de sabor chocolate obtuvo el nivel de agrado más alto y la de sabor plátano alcanzó un nivel significativamente menor.

\section{Resultados de la evaluación en la comunidad}

En el cuadro III se presenta el consumo promedio en gramos y el porcentaje consumido de cada producto por sabor durante la prueba de aceptación. Las diferencias en los consumos de los distintos sabores para los tres productos no fueron significativas. El porcentaje de la porción servida consumida por las mujeres fue más alto que el de la que consumieron los niños.

En el cuadro IV se presenta el consumo promedio en gramos y el porcentaje consumido del producto por sabor durante la prueba de seguimiento. El consumo de papilla fue estadísticamente diferente $(p<0.05)$ entre los diversos sabores, de tal manera que el mayor consumo correspondió al sabor de chocolate, seguido por los de vainilla y plátano. En el caso de la bebida para niños, el grupo que prefirió aquella con sabor a plátano hizo consumos mayores que aquel que eligió el sabor vainilla. Las mujeres hicieron consumos similares para todos los sabores. Para las tres bebidas, independientemente del sabor, se registraron consumos promedio superiores a $75 \%$ de lo ofrecido.

En el cuadro V se muestra el consumo promedio en gramos y el porcentaje consumido del producto en los niños por grupo de edad y en las mujeres embarazadas y en periodo de lactancia, durante la fase de seguimiento. Los lactantes consumieron menores proporciones de las cantidades ofrecidas, tanto de papilla como de bebida, en comparación con los niños destetados $(p<0.05)$. Al comparar las proporciones consumidas del producto según su consistencia, no se observaron diferencias en los niños destetados. Sin embargo, los lactantes consumieron mayor proporción de la papilla del sabor preferido en comparación con los lactantes a quienes se les ofreció el producto en su modalidad de bebida; esta diferencia fue estadísticamente significativa $(p<0.05)$. Lo anterior se reflejó en el consumo notoriamente mayor de calorías de los lactantes que recibieron papilla. En cuanto a la bebida para mujeres, se registraron consumos elevados y no se encontraron diferencias estadísticas entre el consumo de las mujeres embarazadas y el de aquellas que amamantaban.

En la figura 1 se presenta el aporte de energía (Kcal) correspondiente al consumo de los productos evaluados durante la fase de seguimiento. En los tres grupos se observa una tendencia estable en el consumo de los productos; sin embargo, en el caso de los 


\section{Cuadro III}

\section{Promedio de consumo \pm DE de las fórmulas desarrolladas durante la prueba de aCeptación*

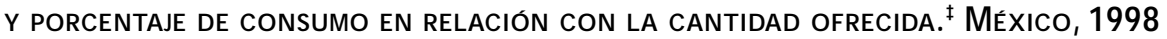

\begin{tabular}{|c|c|c|c|c|c|c|c|}
\hline \multirow[b]{2}{*}{ Producto } & \multirow[b]{2}{*}{$\mathrm{n}$} & \multirow[b]{2}{*}{ Promedio ofrecido $\pm \mathrm{DE}(\mathrm{g})$} & \multicolumn{5}{|c|}{$\begin{array}{c}\text { Promedio de consumo } \pm \mathrm{DE}(\mathrm{g}) \\
\text { (\% consumido) }\end{array}$} \\
\hline & & & Chocolate & Vainilla & Plátano & Natural & Total \\
\hline \multirow[t]{2}{*}{ Papilla } & 116 & $68.7 \pm 1.4$ & $46.4 \pm 18.9$ & $51.7 \pm 18.5$ & $51.5 \pm 16.9$ & ND & $49.9 \pm 18.2$ \\
\hline & & & $(67.6 \%)$ & $(75.3 \%)$ & $(75.0 \%)$ & & $(72.6 \%)$ \\
\hline \multirow[t]{2}{*}{ Bebida niños } & 110 & $191.2 \pm 2.0$ & $122.8 \pm 61.7$ & $131.9 \pm 62.6$ & $134.9 \pm 63.6$ & ND & $129.9 \pm 62.6$ \\
\hline & & & $(64.2 \%)$ & $(69.0 \%)$ & $(70.5 \%)$ & & $(67.9 \%)$ \\
\hline \multirow[t]{2}{*}{ Bebida mujeres } & 128 & $200.2 \pm 2.8$ & ND & $191.9 \pm 28.2$ & $191.7 \pm 26.2$ & $190.0 \pm 32.4$ & $191.2 \pm 29.0$ \\
\hline & & & & $(96.0 \%)$ & $(95.8 \%)$ & $(94.7 \%)$ & $(95.5 \%)$ \\
\hline
\end{tabular}

\section{Cuadro IV}

Promedio de consumo \pm DE de las fórmulas desarrolladas durante dos semanas de seguimiento y PORCENTAJE CONSUMido en Relación CON La CANTIDAd OfReCidA.* México, 1998

\begin{tabular}{|c|c|c|c|c|c|}
\hline \multirow[b]{2}{*}{ Producto } & \multicolumn{5}{|c|}{$\begin{array}{l}\text { Promedio de consumo } \pm \mathrm{DE}(\mathrm{g}) \\
\mathrm{n}(\% \text { consumido })\end{array}$} \\
\hline & Chocolate & Vainilla & Plátano & Natural & Todos los sabores \\
\hline \multirow[t]{2}{*}{ Papilla } & $65.2 \pm 6.4^{\mathrm{a}}$ & $61.2 \pm 10.2^{b}$ & $57.6 \pm 15.8^{c}$ & ND & $60.1 \pm 13.0$ \\
\hline & $9(94.3 \%)$ & $22(88.7 \%)$ & $25(83.4 \%)$ & & $56(87.1 \%)$ \\
\hline \multirow[t]{2}{*}{ Bebida niños } & $145.2 \pm 60.7^{\mathrm{a}, \mathrm{b}}$ & $141.8 \pm 57.1^{\mathrm{a}}$ & $154.7 \pm 50.4^{b}$ & ND & $147.4 \pm 55.8$ \\
\hline & $15(75.8 \%)$ & $19(74.0 \%)$ & $18(80.8 \%)$ & & $52(77.0 \%)$ \\
\hline \multirow[t]{2}{*}{ Bebida mujeres } & ND & $197.3 \pm 7.4$ & $196.5 \pm 4.4$ & $197.4 \pm 4.4$ & $196.9 \pm 8.0$ \\
\hline & & $31(98.5 \%)$ & $53(98.2 \%)$ & $23(98.5 \%)$ & $107(98.3 \%)$ \\
\hline
\end{tabular}

niños estudiados el aporte energético de la papilla fue significativamente mayor que el de la bebida $(p<0.05)$. Las mujeres que tomaron la bebida mantuvieron consumos elevados a lo largo de la prueba de apego al consumo, que se traducen en una ingestión energética cercana a la aportada por la porción ofrecida.

\section{Discusión}

En este estudio se describen las fórmulas y los procesos necesarios para obtener una serie de productos desti- nados a los programas de suplementación alimenticia para niños de 4 meses a 2 años de edad, así como a mujeres embarazadas y en periodo de lactancia, pues constituyen los grupos de población con mayor riesgo de sufrir deterioros en su estado nutricio y, consecuentemente, en su salud. Por otra parte, en la elaboración de estos productos se utilizan ingredientes y se requieren procesos de fabricación comunes y de fácil obtención en México. Se establecieron requisitos con los cuales deberían cumplir los suplementos y que han quedado ampliamente descritos en las diferentes 


\section{Cuadro V \\ Promedio de consumo \pm DE de las fórmulas desarrolladas durante dos semanas de seguimiento POR GRUPO Y PORCENTAJE CONSUMIDO EN RELACIÓN A CANTIDAd OFRECIDA.* MÉXICO, 1998}

\begin{tabular}{|c|c|c|c|c|}
\hline $\begin{array}{l}\text { Grupo } \\
\text { niños }\end{array}$ & $n$ & $\begin{array}{l}\text { Promedio de consumo } \pm \mathrm{DE}(\mathrm{g}) \\
\% \text { consumido }\end{array}$ & $n$ & $\begin{array}{l}\text { Promedio de consumo } \pm \mathrm{DE}(\mathrm{g}) \\
\% \text { consumido }\end{array}$ \\
\hline \multirow[t]{2}{*}{$<2$ años lactantes } & 32 & $56.5 \pm 15.0$ & 26 & $111.7 \pm 54.4^{\mathrm{a}}$ \\
\hline & & $81.8 \%$ & & $58.9 \%$ \\
\hline \multirow[t]{2}{*}{$<2$ años destetados } & 9 & $64.4 \pm 7.6$ & 14 & $174.7 \pm 39.4^{b}$ \\
\hline & & $93.8 \%$ & & $91.0 \%$ \\
\hline \multirow[t]{2}{*}{$>2$ años destetados } & 13 & $65.9 \pm 5.4$ & 14 & $185.7 \pm 20.7^{c}$ \\
\hline & & $95.4 \%$ & & $97.0 \%$ \\
\hline \multirow[t]{2}{*}{ Mujeres embarazadas } & - & ND & 50 & $197.3 \pm 9.9$ \\
\hline & & & & $98.6 \%$ \\
\hline Mujeres en periodo de lactancia & - & ND & 57 & $196.6 \pm 6.0$ \\
\hline \multicolumn{4}{|c|}{$\begin{array}{l}\text { * C antidad ofrecida: papilla } 69 \mathrm{~g} \text {, bebida niños } 191 \mathrm{~g} \text {, bebida mujeres } 200 \mathrm{~g} \\
\text { a,b,c Los supraíndices diferentes entre grupos indican diferencia estadísticamente significativos ( } p<0.05 \text { en la columna) }\end{array}$} & $\begin{array}{l}\text { DE: desviación estándar } \\
\text { N D: producto no disponible }\end{array}$ \\
\hline
\end{tabular}

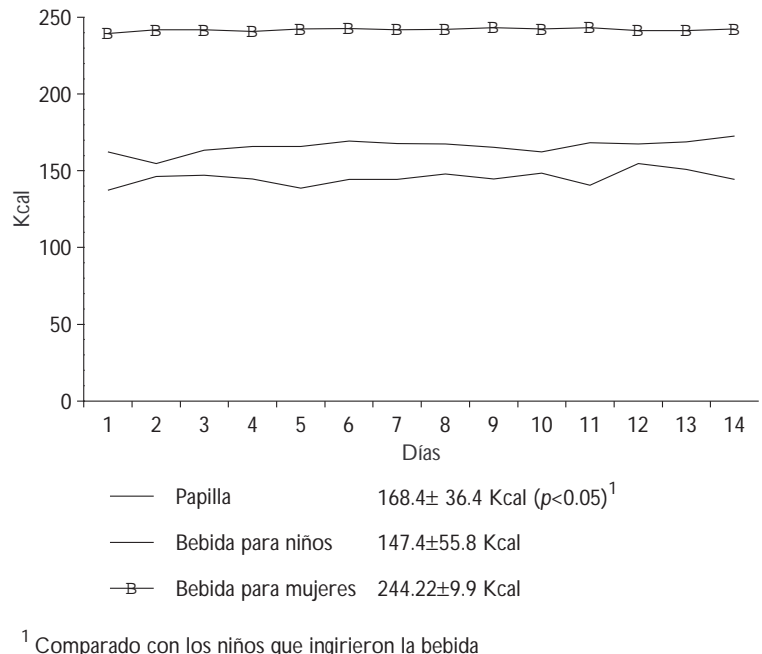

Figura 1. Promedio del consumo diario de energía DURANTE DOS SEMANAS

secciones de este documento. Por último, durante el desarrollo de los suplementos se buscó la forma más fácil y barata de producirlos. Las evaluaciones sensoriales y en comunidad demostraron que los productos cumplieron con los propósitos para los cuales se desarrollaron.
La aceptación de los productos en sus distintos sabores fue elevada desde que se consumieron por primera vez. Los niños tomaron más del $65 \%$ y las mujeres más del 95\% de la ración que se les ofreció. La cantidad que consumieron las mujeres fue prácticamente la totalidad de la cantidad que se les ofreció, si se descuenta el residuo que se adhiere al recipiente. Las proporciones consumidas fueron elevadas, considerando que en la prueba de aceptación aún no había habituación a la bebida. Durante la prueba de seguimiento en la que se evaluó el sabor de preferencia de los distintos productos durante dos semanas, los consumos aumentaron en los niños a valores de $75 \%$ o más de la ración servida. El aumento en los consumos durante la prueba de seguimiento se debe, probablemente, a que durante la prueba de seguimiento se evaluó el sabor preferido, mientras que en la prueba de aceptación los niños y las mujeres tuvieron contacto con el producto por primera vez. Cabe observar que en el caso de las mujeres la aceptación y el apego al consumo de la bebida fueron satisfactorios desde el inicio del estudio, destacando los elevados niveles de consumo tanto de las embarazadas como de las mujeres en periodo de lactancia.

Al menos durante las dos semanas de seguimiento no hubo evidencia de pérdida de interés por los productos. En dicho periodo los niños consumie- 
ron en promedio $168 \mathrm{Kcal}$ de papilla y $147 \mathrm{Kcal} /$ día de bebida; ambas cantidades son superiores a los consumos que se indican en estudios controlados de suplementación alimentaria y que han documentado efectos importantes en el crecimiento. Así por ejemplo, en un ensayo que desarrolló el Instituto de Nutrición de Centroamérica y Panamá (INCAP) se encontró un efecto de la suplementación de entre dos y tres centímetros en longitud de niños que recibieron una bebida entre los tres meses y los tres años de vida. El consumo promedio de energía procedente de la bebida fue de 90 a $100 \mathrm{Kcal}^{.5}$ En nuestro estudio, los consumos observados tanto en el grupo que recibió papilla como en el que recibió bebida se encuentran muy cercanos al máximo promedio observado en el estudio del INCAP, que fue de $157 \mathrm{Kcal} /$ día a los 30 meses, en una de las comunidades donde se distribuyó el suplemento. ${ }^{6}$ Así entonces, las cantidades de energía que consumieron los niños del presente estudio son suficientes para lograr impactos en el crecimiento en poblaciones con elevada prevalencia de desmedro, con la ventaja de que los productos aportaron cantidades adicionales de vitaminas y minerales indispensables para el desarrollo.

En el estudio guatemalteco al que se ha hecho referencia, las mujeres embarazadas recibieron la misma bebida ofrecida a los niños. Se documentó un impacto positivo en el peso de los recién nacidos de mujeres que recibieron cantidades significativas de la bebida durante los dos últimos trimestres del embarazo (111 o más calorías/día). La magnitud del efecto del suplemento en el peso al nacer fue de $117 \mathrm{~g}$, similar al de otros ensayos controlados de suplementación alimenticia y que se considera biológicamente importante. El promedio diario de consumo del suplemento en el grupo en el que se demostró este impacto fue de 233 Kcal/día, cantidad inferior a la que consumieron, en promedio, las mujeres del presente estudio. Así entonces, queda demostrado que la energía que consumieron las mujeres de este estudio es suficiente para lograr impactos en peso al nacer en poblaciones desnutridas.

Una vez que los niños y las madres estudiadas seleccionaron el sabor preferido de los suplementos, el consumo de los mismos se incrementó en comparación con el del inicio del estudio. Es interesante observar que el consumo de un sabor específico durante la fase de aceptación no es el mismo que el registrado en un periodo más largo. Tal es el caso de la papilla sabor chocolate, cuyo consumo aumentó en forma importante durante la fase de seguimiento.

Al comparar la cantidad consumida durante el seguimiento por grupo de edad se observa que, particularmente en el caso de la bebida, los lactantes me- nores de dos años hicieron consumos inferiores a los de los niños de los grupos de mayor edad, incluyendo aquellos con desnutrición. Asimismo, en la mayoría de los casos se encontraron consumos de energía más elevados del producto en su modalidad de papilla.

En el caso de las mujeres es interesante observar que la aceptación y el apego al consumo de la bebida fueron satisfactorios desde el inicio del estudio, y que el consumo fue elevado tanto entre las embarazadas como entre las que se encontraban en periodo de lactancia.

Así entonces, se concluye que el producto en sus modalidades de papilla y de bebida tiene una buena aceptación entre los grupos estudiados, al inicio de su administración y por un lapso de dos semanas. Asimismo los sabores que se evaluaron se consumieron en un nivel que garantizó el aporte de energía y nutrimentos esperado. Por otra parte es necesario destacar que dos semanas son insuficientes para evaluar el consumo de largo plazo, de tal manera que es importante hacer estudios de mayor duración en el futuro.

\section{Agradecimientos}

Los autores agradecen el apoyo económico de la Secretaría de Salud, del Instituto Nacional de la Nutrición Salvador Zubirán y del Instituto Nacional de Salud Pública para la realización de los estudios descritos.

\section{Referencias}

1. Instituto $\mathrm{N}$ acional de la $\mathrm{N}$ utrición Salvador Zubirán. Encuesta $\mathrm{N}$ acional de Alimentación en el Medio Rural EN AL-89. México, D.F.: IN N SZ/C omisión N acional de Alimentación, 1990.

2. Madrigal H, Batrouni L, Ramírez D, Serrano L. Cambio en el consumo de alimentos en México. Rev Invest Clin 1986;385:33-39.

3. Rosado JL, Bourges H, Saint-Martin B. D eficiencia de vitaminas y minerales en México. Una revisión crítica del estado de la información: II. Deficiencia de vitaminas. Salud Publica Mex 1995;37:452-461.

4. Rosado JL, Bourges H, Saint-Martin B. D eficiencia de vitaminas y minerales en México. Una revisión crítica del estado de la información: I. D eficiencia de minerales. Salud Publica Mex 1995;37:137-139.

5. Martorell R. 0 verview of long-term nutrition intervention studies in Guatemala, 1968-1989. Food N utr Bull 1993;14:270-277.

6. Mora JO, Herrera MG, Suescun J, D e N avarro L,W agner M.The effect of nutritional supplementation on physical growth of children at risk of malnutrition. Am J Clin N utr 1981;34:1885-1892.

7. Balachander J.Tamil N adu's successful nutrition effort. En:Rodhe J, C hatterjee M, Morley D, comp. Reaching health for all. N ueva D ehli: India: 0 xford University Press, 1993:159-184.

8. Scrimshaw N S. Programs of supplemental feeding and weaning food development. En: Scrimshaw N S, W allerstein MB, ed. Nutrition policy implementation. Issues and experience. Nueva York: Plenum Press, 1982;101-111. 
9. N aranjo A, González A, Chávez A. Evaluación de un paquete de nutrición para mejorar la salud infantil. Metodología y cambios en el patrón de ablactación y destete. Rev Invest Clin 1986;38S:137-146.

10. Rivera J, Long K, González-Cossío T, Parra S, Rivera M, Rosado JL. $N$ utrición y salud:un menú para la familia. En:C uadernos de Salud. Problemas Pretransicionales. México, D.F.: Secretaría de Salud, 1994.

11. Flores M, Melgar H, Cortés C, Rivera M, Rivera J, Sepúlveda J. Consumo de energía y nutrimentos en mujeres mexicanas en edad reproductiva. Salud Publica Mex 1998;161-171.

12. Lisker R, López-Habib G, D altabuit M, Rostenberg I. Lactase deficiency in a rural area of Mexico.Am J C lin N utr 1974;27:756-759.

13. C ifuentes GE, Flores SJJ, Limón GN E. D eficiencia de lactasa intestinal en un pueblo nahua: alternativas para los programas de intervención nutricional en la región. Rev Invest Clin 1985; 37:311-315.

14. Palma M, Rosado JL, López P, G onzález C,Valencia ME. Intolerancia a la lactosa. Su definición, su prevalencia en México y sus implicaciones en el consumo de leche. Rev Invest Clin 1996; 48S:25-31.
15. López P, Rosado JL, Palma M, González C, Valencia ME. Mala digestión de lactosa. Su definición, su prevalencia en México y sus implicaciones en el consumo de leche. Rev Invest Clin 1996; 48S:15-22.

16. Rosado JL, González C,Valencia ME, López P, Palma M, López B et al. Lactose maldigestion and milk intolerance: $A$ study in rural and urban Mexico using physiological doses of milk.J N utr 1994;124:1052-1059. 17. Rosado JL, Lisker R. Implicaciones de la mala digestión y de la intolerancia a la lactosa en la salud pública. Rev Inv C lin 1996;485:87-88.

18. Rosado JL. Lactose digestion and maldigestion: Implications for dietary habits in developing countries. Nutr Res Rev 1997;10:137-149.

19. Stone H, Sidel JL. Sensory evaluation practices. Food Science and Technology. A series of monographs. N ueva York:Academic Press, 1985.

20.0 rganización Mundial de la Salud. Medición del cambio del estado nutricional. Ginebra: O MS, 1983. 